\title{
Retrospective Study of Common Bile Duct Injury after Laparoscopic Cholecystectomy
}

\section{A.A.Salem, R.S.Salama, M.E.Abd El-Latif and A.E.El-Gazzar}

General Surgery Dept., Faculty of Medicine, Benha Univ., Benha, Egypt

E-Mail: amrelgazzar22@gmail.com

\begin{abstract}
Laparoscopic cholecystectomy is one of commonest laparoscopic surgeries but carries out serious complications, one of them with high morbidity and mortality which is Bile Duct Injury. Biliary leak, biliary peritonitis and obstructive jaundice presents BDI with different management planes from ERCP and stent application up to abdominal exploration and roux-eny hepatico-jeujenostomy to restore bile system continuity, this fact drove the attention of healthcare institutes to this problem. Is to assess different types of Bile duct injuries (BDI) after laparoscopic cholecystectomy and the outcome of different surgical procedures and recent radiological interventional procedures. This is a retrospective study carried on 50 patients underwent laparoscopic cholecystectomy and complicated by bile duct injury in benha university hospitals, after approval of local ethics committee, all patients included in the study were informed about the procedure and a written consent was obtained from every patient before carrying the different surgical procedures and recent radiological interventions. The study was done from January 2004 till January 2020. BDI during laparoscopic cholecystectomy occur more frequently when compared to open surgery. They occur twice to three times, more frequently than after an open surgery. Among early presentations is bile leak which occurred in (56\%) who present early within first week. Different plans of management which differs from radiological intervention with (14\%) up to abdominal exploration with (70\%). Increased incidence of bile duct injury with laparoscopic cholecystectomy more than open cholecystectomy. Over confidence of some surgeons can cause serious damage. Roux en y Hepatico-jeujonostomy is the preferred surgical procedure. Intervention radiology plays important role in diagnosis and management of BDI.
\end{abstract}

Keywords: ERCP, Biliary-enteric anastomosis, Obstructive Jaundice, Bile leakage.

\section{Introduction}

Laparoscopic cholecystectomy is widely used as the standard operation for benign GB disease that require Cholecystectomy as laparoscopic cholecystectomy but despite the progress achieved IBDI after laparoscopic cholecystectomy is considered a serious situation with high morbidity and non-negligible low mortality. Laparoscopic cholecystectomy (LC) has led to a decrease postoperative pain and hospital stay and allowed early return to work and daily activity, but unfortunately has increased some complications such as bile duct injury from $0.3 \%$ to $1.2 \%$ [14]

Bile duct injury results from bad identification of anatomy of biliary tree by the surgeon and orientation of anatomical variations of the biliary tree, recurrent attacks of acute inflammation and over confidence of some surgeons[17].

Callot's triangle is the most important anatomical and intraoperative surgical land mark, so for proper safety and success of the operation, surgeon must have to identify targeted anatomical structures of the gall bladder, patients with bile duct injury mostly presents with post-operative bile leak or obstructive jaundice according to the cause either ligature or injury of bile ducts [6].

Biliary leak is the commonest complications of bile duct injury after laparoscopic cholecystectomy with early presentation post operatively while stenosis or stricture is considered late complication but both stenosis, stricture or occlusion present by obstructive jaundice. The best way to treat bile duct injury is to avoid the injury by assessment of bile duct anatomy, critical view of safety approach in laparoscopic cholecystectomy intraoperative cholangiography and laparoscopic ultrasonography should be performed routinely to prevent bile duct injury and increase patient safety and postoperative condition [2].

Obstructive jaundice is also a serious complication but is presented later than bile leakage and can be caused by thermal injury, causing stricture, up to misidentification of CBD with wrong clipping of CBD. For repair it needs re-fashioning and biliary enteric anastomosis depending on level of injury [2].

Currently the standard management of suspected biliary leak after laparoscopic cholecystectomy remains to be determined and clinical and imaging findings are taken into account in such patients. Also in an emergency setting noninvasive imaging would be beneficial for planning further management [3].

Many methods are developed to repair biliary injury as: t-tube insertion, biliary stenting, hepaticojeujnostomy or roux-en-y anastomosis, all these are decided according to type of injury, level of injury and general condition of the patient, high level of scrutiny in the intraoperative and immediate postoperative phases are required, and also a multidisciplinary approach between surgeons, radiologists and gastroenterologists to give the patient the best initial diagnosis, the best treatment options and best management of complications [16].

\section{Patients and methods}

This is a retrospective study carried on 50 patients underwent laparoscopic cholecystectomy and complicated by bile duct injury in benha university hospitals, after approval of local ethics committee, all patients included in the study were informed about the procedure and a written consent was obtained from every patient before carrying the different surgical procedures 
and recent radiological interventions. The study was done from January 2004 till January 2020.

\subsection{Inclusion criteria}

Any patient underwent laparoscopic cholecystectomy and complicated by bile duct injury and in this study we had 50 patients with:

1- Age: From 20 to 74 years old.

2- Sex: Both sex male and female.

3- BDI after laparoscopic cholecystectomy.

4- Patients admitted to benha university hospital for L.C.

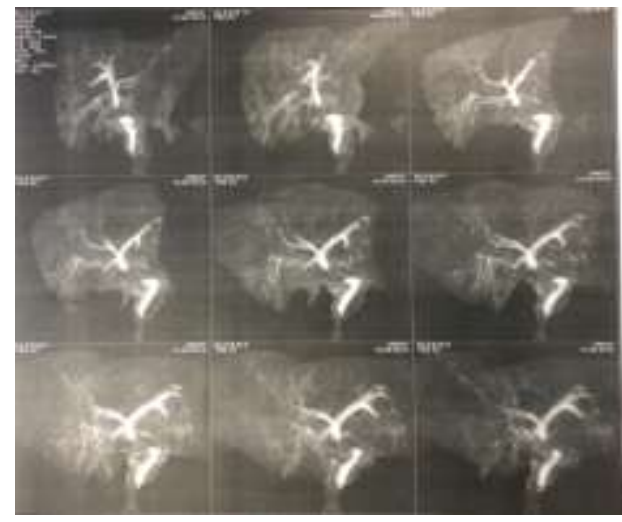

Fig (1) PTC showing complete CBD occlusion 1 $\mathrm{cm}$ from bifurcation.

\subsection{Preoperative work up}

\section{History and clinical examination}

Age, sex, occupation, smoking history, presentation and time of presentation, timing of primary surgery (L.C.), History of abdominal operations or recurrent of acute cholecystitis, history of chronic diseases as HTN, DM, CKD, CLD or cardiac diseases, any known congenital anomalies of the patient, informed written consent.

\section{Routine laboratory work-up}

CBC, complete LFT (SGPT, SGOT, S. Bilirubin, Alk.Ph., S. Alb., GGT), KFT, PT, PTT, INR. Radiological investigations: ECG, CXR, Pelviabdominal ultrasound, CT abdomen and MRCP. Evaluation of primary surgery (L.C.), drain output if present, scars of previous abdominal operations.

\section{Preoperative preparation}

The successful biliary surgical repair should be based on SNAP principle as follow:

( $\mathbf{S}=$ stabilization and sepsis control, $\mathbf{N}=$ Nutrition, $\mathrm{A}=$ Anatomy of bile system, $\mathrm{P}=$ Plan of management)

- Preparation of packed RBCs and fresh frozen plasma.

- Fasting period of 6-8 hours.

\subsection{Exclusion Criteria}

In this study, there are no exclusion criteria but the following patients could be excluded:

1. Patients with lost follow up.

2. Patients who died due to either sepsis and peritonitis or any other cause as pulmonary embolism or heart failure.

\subsection{Presentation}

Patients were divided into two main groups according to presentation after primary surgery as:

- Group A: presented by obstructive jaundice

- Group B: presented by biliary leak, bile collection, fever, abdominal pain, nausea and vomiting.

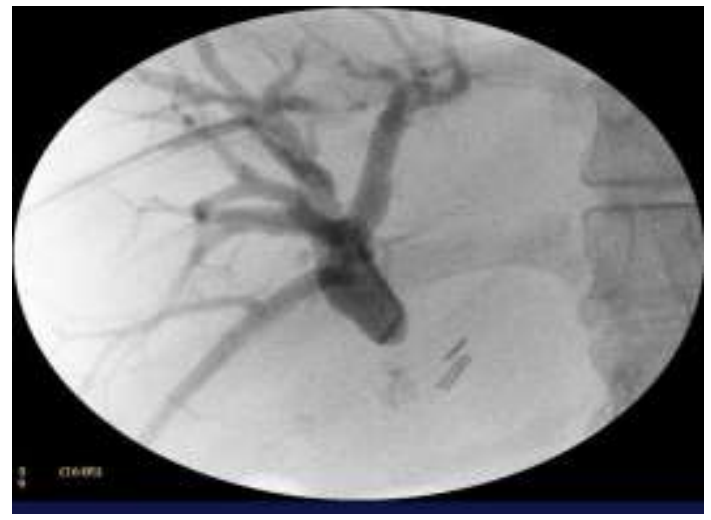

Fig (2) PTC showing LC clips and occlusion of CBD

- Anesthesia: general anesthesia with endotracheal intubation

- Foley's catheter.

- Nasogastric tube application.

Timing of management

Timing of interference depends on the time of diagnosis of BDI which is either:

- Shortly post-operative within one week by biloma, bile leak through drain if present or biliary peritonitis.

- A relatively longer post-operative by jaundice.

So, in case of biloma and the patient is hemodynamically stable, Pig-tail drain could be enough and avoid surgery. In case of bile leak through drain with low out-put, conservative treatment should be considered. In case of biliary peritonitis, urgent abdominal exploration is mandatory after stabilization of the patient by SNAP principle with the final step which is the Plan of management as follow:

Non-surgical procedures:Ultrasound guided aspiration or pig-tail drain insertion by intervention radiology team, ERCP with stent application and endoscopic sphincterotomy by gastro-enterologis. 


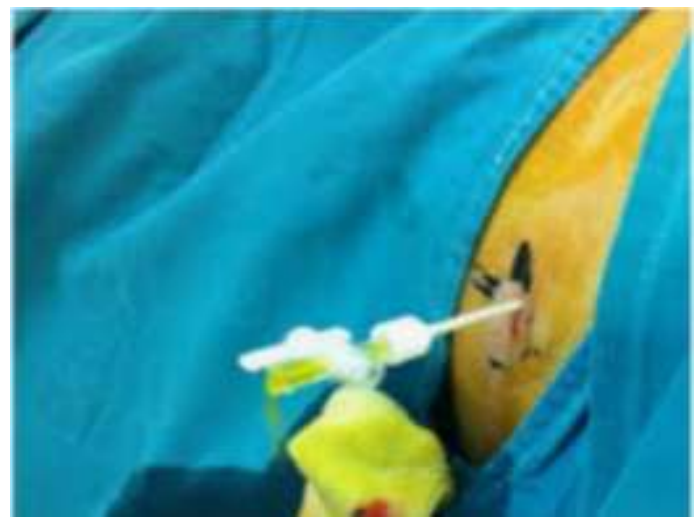

Fig (3) Pig-tail drain applied into biloma.

\subsection{Surgical techniques}

The procedure was done under general anesthesia with endo tracheal intubation. Skin disinfection starting from nipple line till thigh was done, followed by draping. Mostly midline incision with extended right subcostal incision, in midline incision with extended right subcostal incision. Approaching biliary system and performing the planned operation according to injury as direct biliary enteric anastomosis, Roux-en-y hepaticojeujonostomy, cystic duct closure and repair over T-tube insertion.

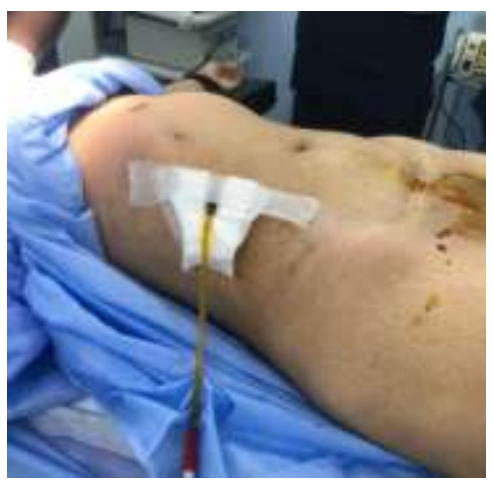

Fig (4) Bile leak in drain after L.C.

- Cystic duct closure: in case of cystic duct leak not treated by ERCP, patient need just cystic stump reclipping either by laparoscope in case of no peritonitis or open surgery in case of peritonitis to do peritoneal lavage and drain application.

- Repair over T tube drain: after approaching CBD and exploring site of injury, longitudinal incision running distally towards duodenum in the anterior surface of CBD though which $\mathrm{T}$ tube drain is inserted to apply by the two short limbs are up and down in CBD and the long limb is coming through the made opening out to the skin over collecting

- In case of severe peritonitis: the goal is to eradicate sepsis and delay repair so first step is to exteriorize and drain bile system outside body and planned repair later after eradication of sepsis.

- Hepatico-jeujenostomy: In case of high injury or sectorial injury, Roux-en-Y hepatico-jejunostomy (RYHJ) is currently considered as the definitive treatment for iatrogenic bile duct injuries and the principal representative of biliary diversion procedures.

After careful dissection and division of the extrahepatic, hilar, or intrahepatic bile duct(s) the Roux-
en-Y jejunal limb is then prepared by transecting the jejunum around $20-30 \mathrm{~cm}$ distal from the Treitz ligament and then brought in a retro-colic fashion, right of the middle colic vessels, to the right upper abdomen. care must be taken to ensure a tension-free jejunal limb with sufficient length. A small orifice $(5 \mathrm{~mm})$ at the antimesenteric side of the Roux-limb which should always be much smaller than the width of the hepatic duct. The mucosa of the intestinal orifice is slightly inverted, using four PDS 5-0 interrupted stitches in a "crosswise" fashion, in order to create a mucosa-to-mucosa anastomosis.

For the construction of our single-layer, end-to-side HJ we use 4-0 to 6-0 PDS interrupted sutures. The first two sutures are placed in the left corner of the jejunum and the bile duct. The needles are passed through the bile duct from outward to the inside and then through the jejunum from the inside outwards. The jejunal limb is then gently pushed down to the hepatic duct and the sutures are tied. All the passing of the stiches takes a good amount of sero-muscular part of the small bowel but not the mucosa, helping the mucosa to be inside the 
bile duct and so to complete a mucosa-to-mucosa anastomosis.

After the completion of the anastomosis, control for bile leaks (if present) should be performed. When a PTBD is in place, a "white-test" with propofol or lipiodol can be made in order to check the patency and the integrity of the anastomosis. The mean operative time of the technique is 74 minutes. We strongly believe that the key-points to the long-lasting results of this technique are the prevention of ischemia, the avoidance of bile leak, and the mucosa-tomucosa anastomosis.

Lastly, the final anastomosis is jejuno-jejunostomy to maintain continuity of GIT by side to end anastomosis either by stapler or hand sewing technique. After completion of operation, make sure that no leak or any intra-peritoneal collection after lavage and apply drains at site of anastomosis and another pelvic drain.

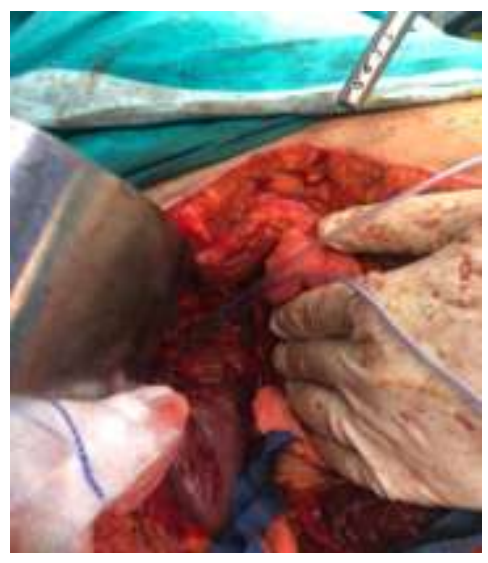

Fig (5) 2 drains each in right and left hepatic ducts

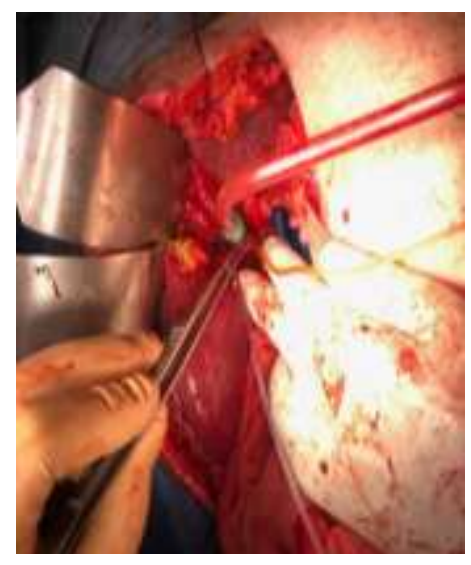

Fig (6) Bile coming out through drain in left hepatic duct

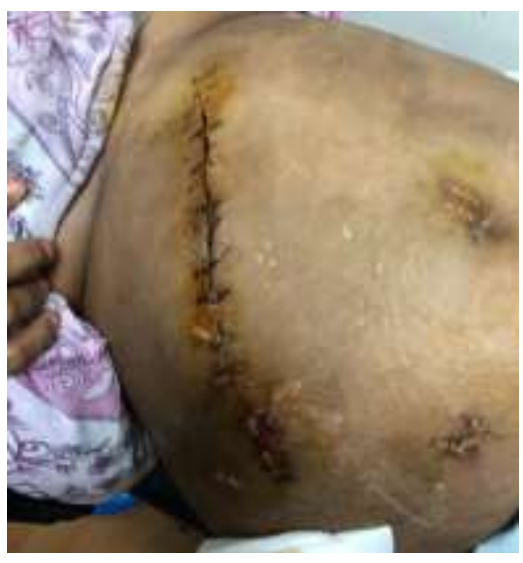

Fig (7) Follow up of scar after 2 weeks of opeartaion

\subsection{Postoperative care and follow up}

All patients are post operatively are kept either in ward or ICU according to patient's general condition with daily assessment with ultrasound, CBC, abdominal examination and drain output to exclude further intraperitoneal collection. Drains are removed when they stop to drain except for pig tails and T-tubes are removed after 4-6 weeks, start oral intake when intestine restore its normal movement and auscultated, outpatient clinic follow up of left drains and abdominal examination.

Don't rush for discharging the patient until he start oral feeding and make sure that there is no residual or recollection intra-abdominally.

The interventional radiologist is involved in both diagnosis and treatment of patients affected by post-

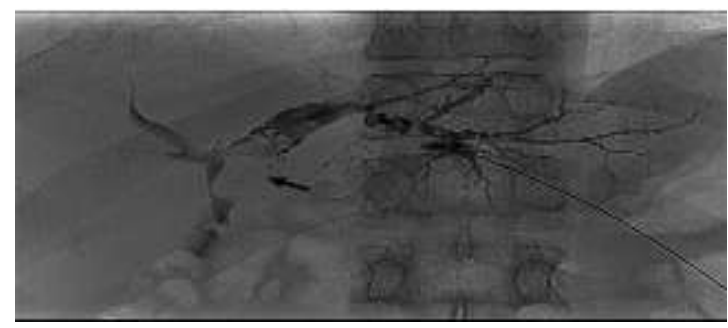

Fig (8) PTC showing multiple left intrahepatic bile duct stones, a large cone shaped common hepatic duct stone and hepatico-jejunal anastomotic stenosis (arrow) (Akgür et al., 2015) surgical biliary tract stenosis or biliary leakage. The etiology of benign strictures of the biliary tract is: iatrogenic, liver transplantation or chronic pancreatitis. The last 2 causes, however, are less common and do not need an interventional radiological treatment. Historically, the incidence of iatrogenic lesions in the biliary ducts increased significantly after the introduction of laparoscopic procedures, mainly laparoscopic cholecystectomy. In literature, the complication rate ranges between $0 \%$ and $4 \%$ with an average of $0.5 \%$. In $75 \%$ of cases, the lesions occur in correspondence of the main ducts and the main confluences; in $25 \%$ of cases the cystic duct or minor ducts are involved [11].

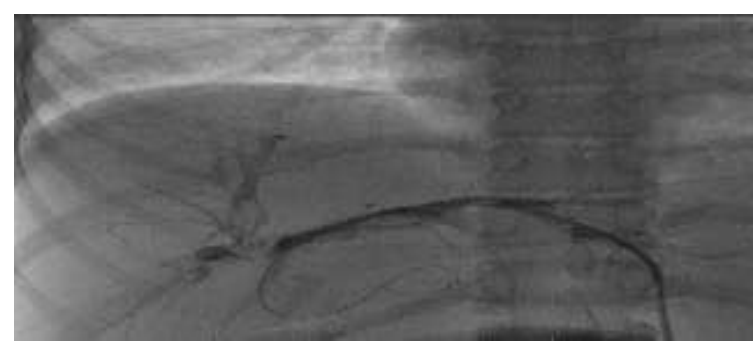

Fig (9) PTC showing passage of guide from bilioenteric anastomotic stenosis. (Akgür et al., 2015)

The study includes 50 patients undergone laparoscopic cholecystectomy and complicated by biliary duct injuries and 
the results of the current study are summarized in the following tables and figures.

Table (1) shows the descriptive data of 50 patients undergone L.C. complicated by BDI according to their sex as we got 31 females and 19 males with higher incidence with female sex as they are higher incidence for L.C.

Table (2) shows the descriptive data of 50 patients undergone L.C. complicated by BDI according to presentation either obstructive jaundice or bile leak.

Table (3) shows the descriptive data of 50 patients undergone L.C. complicated by BDI with possible risk factors as acute cholecystitis, morbid obesity, previous abdominal operation, chronic liver disease and patients with undetected risk factors with higher incidence with acute cholecystitis with second higher incidence of morbid obesity.

Table (4) shows the descriptive data of 50 patients undergone L.C. complicated by BDI according to time of presentation with higher incidence of delayed presentation more than a week by present of $62 \%$ which counts for about 31 patients of 50 .

Table (5) shows the descriptive data of 50 patients undergone L.C. complicated by BDI and radiological investigations needed to diagnose type of injury and as we see all patients made PAUS as it is cheap noninvasive maneuver followed by ERCP as it can be used as diagnostic and therapeutic maneuver and proved high success rate and substituted surgery in many cases.

Table (6) show the descriptive data of 50 patients undergone L.C. complicated by BDI and the type of injury according to Strasberg classification of BDI with the higher incidence to types E4 and E5.

Table (7) show the descriptive data of 50 patients undergone L.C. complicated by BDI and the different management planes.

Table (1) Sex of group cases.

\begin{tabular}{lc}
\hline Sex & No. of patients $(\%)$ \\
\hline Female & 31 patients $(62 \%)$ \\
Male & 19 patients $(38 \%)$ \\
\hline
\end{tabular}

Table (2) Presentation of patients.

\begin{tabular}{lc}
\hline Presentation & No. of patients $(\%)$ \\
\hline Biliary leak, & 28 patients $(56 \%)$ \\
Obstructive jaundice & 22 patients $(44 \%)$ \\
\hline
\end{tabular}

Table (3) Risk factors of group cases.

\begin{tabular}{lc}
\hline Risk factor & No. of patients (\%) \\
\hline Acute cholecystitis & 15 patients $(30.0 \%)$ \\
Morbid obesity & 11 patients $(22.0 \%)$ \\
Previous abdominal operation & 7 patients $(14.0 \%)$ \\
Chronic liver disease & 6 patients $(12.0 \%)$ \\
No risk factors detected & 11 patients $(22.0 \%)$ \\
\hline
\end{tabular}

Table (4)Time of presentation of group cases.

\begin{tabular}{lcc}
\hline Time of presentation & No. of patients & $\%$ \\
\hline Presented in first week postoperative & 28 patients & 56 \\
Presented after 2 weeks postoperative & 22 patients & 44 \\
\hline
\end{tabular}

Table (5) Investigations done to different group cases.

\begin{tabular}{lc}
\hline Radiological investigation & No. of patients $(\%)$ \\
\hline Pelvi-abdominal ultrasound $(\mathbf{U} / \mathbf{S})$ & 50 patients $(100.0 \%)$ \\
CT scan & 9 patients $(18.0 \%)$ \\
MRCP & 5 patients $(10.0 \%)$ \\
ERCP & 36 patients $(72.0 \%)$ \\
\hline
\end{tabular}

Table (6) Type of BDI according to Strasberg classification.

\begin{tabular}{lc}
\hline Type of injury & No. of patients $(\%)$ \\
\hline A & 3 patients $(6 \%)$ \\
B & 3 patients $(6 \%)$ \\
\hline Table (6) Continue & 3 patients $(6 \%)$ \\
\hline C
\end{tabular}




\begin{tabular}{lc}
\hline D & 8 patients $(16 \%)$ \\
E2 & 6 patients $(12 \%)$ \\
E4 & 15 patients $(30 \%)$ \\
E5 & 12 patients $(24 \%)$ \\
\hline
\end{tabular}

Table (7) Different management planes of BDI.

\begin{tabular}{lc}
\hline Management & No. of patients(\%) \\
\hline ERCP, stent application & 9 patients $(18 \%)$ \\
Cystic duct closure & 3 patients $(6 \%)$ \\
Roux-en-y hepaticojeujonostomy & 35 patients $(70 \%)$ \\
CBD repair over T-tube & 3 patients $(6 \%)$ \\
\hline
\end{tabular}

\section{Discussion}

Despite the progress achieved, Iatrogenic bile duct injury (IBDI) after laparoscopic cholecystectomy (LC), being one of the most common performed surgical procedures, remain a substantial problem in gastrointestinal surgery. The most important aspect regarding this issue is the prevention of IBDI during

index cholecystectomy. Since the introduction of LC more than 20 years ago, the rate of BDI does not seem to be substantially changed, even if some researchers report a trend toward a decrease [9].

Bile duct injury results from bad identification of anatomy of biliary tree by the surgeon and orientation of anatomical variations of the biliary tree, these biliary tree anatomical variations occur in about $25 \%$ of patients with aberrant right hepatic ducts (RHD) being the most common, another common variation is low insertion of a right sectorial hepatic duct into the common hepatic duct or the cystic duct increases the risk of injury during both open and laparoscopic cholecystectomy, So detailed knowledge of all possible anatomical variations is crucial for surgeons to avoid such complications [17].

Biliary leak and stricture are the commonest complications of bile duct injury after laparoscopic cholecystectomy with early presentation post operatively while stenosis is considered late complication, but both stenosis or stricture or occlusion present by obstructive jaundice, Investigations needed for assessing biliary injury: serum bilirubin, ALP, TLC in addition to radiology: abdominal ultrasonography and Intraoperative cholangiogram or postoperative ERCP, thermal injury is considered type of bile duct injury causing stricture of bile duct with late either bile leak or obstructive jaundice, Along postoperative course and biliary leakage after removing t-tube are associated with external drainage [16].

A study on 43 patients undergone laparoscopic cholecystectomy and complicated by bile duct injury and by analysis of the presentation of the patients found that 18 patients presented by obstructive jaundice and 25 patients presented by bile leak and biliary peritonitis, so slightly elevated incidence of obstructive jaundice than bile leak [5].

A study performed on 32 patients undergone laparoscopic cholecystectomy and complicated by bile duct injury and found that 12 patients presented by obstructive jaundice and 20 patients presented by bile leak, vomiting, abdominal pain and peritonitis, so increased incidence of obstructive jaundice than bile leak [1].

In our study a descriptive data of the 50 patients complicated by bile duct injury after laparoscopic cholecystectomy, 22 patients presented by obstructive jaundice and 28 patients presented by bile leak, vomiting, abdominal pain and fever, so also our study find that obstructive jaundice is slightly more common than bile leak presentation.

A study performed on 67 patients and by analysis with the possible risk factors found that 16 patients was operated on during acute cholecystitis, 10 patients had history of previous abdominal operation (laparotomy), 4 patients had morbid obesity, 3 patients had cirrhotic liver and 13 patients no risk factor could be detected, so the most common risk factor is acute cholecystitis [15].

An old retrospective study of IBDI after laparoscopic cholecystectomy reported that between many risk factors, the commonest risk factors among them are recurrent attacks of acute inflammation causing dense scaring and morbid obesity [13].

In our study a descriptive data of the 50 patients complicated by bile duct injury after laparoscopic cholecystectomy and analysis of risk factors, 15 patients operated on during acute cholecystitis, 11 patients with morbid obesity, 7 patients with pervious abdominal operation (laparotomy), 6 patients with cirrhotic liver and 11 patients proved no risk factor detected, so commonest risk factor is cholecystitis as it causes dense scarring and amalgamated tissue distorting normal anatomy making the injury more possible. Previous abdominal operations increase intraperitoneal adhesions and subsequently increase incidence of BDI. Cirrhotic liver cause fibrosis and may cause distortion of normal anatomy increasing incidence of BDI. Patients with no RF detected may be due to unexperienced surgeon, lake of facilities or technical problem as disused before.

A study of 59 patients undergone laparoscopic cholecystectomy and complicated by bile duct injury with screening of time of presentation 6 patients discovered intra-operatively, 33 patients presented with in two weeks post-operative and 20 patients presented more than two weeks post-operatively, so delayed presentation must be considered as shown that most of cases are presented lately ${ }^{[\mathbf{1 2}] \text {. }}$

A study on 43 patients undergone laparoscopic cholecystectomy and complicated by bile duct injury with screening of time of presentation 6 patients diagnosed intra-operatively, 17 patients presented with in 
two weeks post operatively and 19 patients presented late more than two weeks of primary surgery, so delayed presentation must be considered as shown that most of cases are presented lately [5].

In our study a descriptive data of the 50 patients complicated by bile duct injury after laparoscopic cholecystectomy and screening of time of presentation found 28 patients presented with in first week postoperatively and 22 patients presented lately after the first week of primary operation, so delayed presentation must be considered and also strict post-operative follow up for early detection of bile leak.

Reported study on 32 patients undergone laparoscopic cholecystectomy and complicated by bile duct injury with needed radiological investigations as all 32 patients made pelviabdominal ultrasonography, 7 patients needed CT abdomen, 2 patients needed MRCP and 23 patients needed ERCP, so it indicates that pelvi-abdominal ultrasonography is mandatory as it is cheap and non-invasive followed by ERCP as it can be used as diagnostic and curative in many cases [1].

In our study a descriptive data of the 50 patients complicated by bile duct injury after laparoscopic cholecystectomy with screening of radiological investigations needed, all 50 patients done pelvi-abdominal ultrasonography, 9 patients done CT abdomen, 5 patients done MRCP and 36 patients done ERCP, so it indicates that pelvi-abdominal ultrasonography is mandatory as it is cheap and non-invasive followed by ERCP as it can be used as diagnostic and curative in many cases.

To many classification systems are used to classify bile duct injury but in our study we will depend on Strasberg classification as it is more accurate than Bismuth classification so according to Strasberg classification we reported 3 patients with type A (cystic duct leak), 3 patients with type B injury (occlusion of aberrant right hepatic duct), 3 patients with type $C$ injury (transection of aberrant bile duct), 8 patients with type D injury (lateral injury to major bile duct), 6 patients with type E2 (high CHD stricture), 15 patients with type E4 (separated right and left hepatic ducts), 12 patients with type E5 (as type 4 plus separation of aberrant right duct).

A study on 32 patients undergone laparoscopic cholecystectomy and complicated by bile duct injury with screening of different management plans, 2 patients went through conservative treatment and follow up of sub-hepatic drain, 7 patients went through ERCP, stent application and endoscopic sphincterotomy, 4 patients went through abdominal exploration with CBD repair over T-tube, 2 patients went through abdominal exploration and cystic duct closure, 16 patients went through Roux-en-y hepaticojeujonostomy. So the plan of management differs according to type of injury as shown in chapter of results [1].

In our study a descriptive data of the 50 patients complicated by bile duct injury after laparoscopic cholecystectomy with viewing of possible management plans as shown 9 patients done ERCP with stent application, 3 patients done abdominal exploration and cystic duct closure, 35 patients needed Roux-en-y hepaticojeujonostomy, 3 patients went through exploration and $\mathrm{CBD}$ repair over T-tube.

A study performed on 70 patients undergone laparosopic cholecystectomy and complicated by bile duct injury with concentration on role of intervention radiology, founded that 17 cases treated by PTD and balloon dilatation and 13 cases treated by ERCP with stent application with good outcomes and follow up [10].

We found that 6 patients who underwent abdominal exploration for Roux-en-y hepatico-jeujonostomy presented later with obstructive jaundice, by investigations revealed 5 cases with anastomotic stricture and 1 case with stone formation on top of anastomotic site, so here comes the role of intervention radiology, these 5 cases were treated by PTD and balloon dilatation, the case with stone ERCP were done, stone extracted and stent applied.

\section{Conclusion}

- Our study showed that although increased incidence of bile duct injury with laparoscopic cholecystectomy more than open cholecystectomy but injury can be avoided by many ways and different management plans are applied according to type of injury. So we can conclude that the most important issue is prevention of Injury itself.

- Increased experience and over confidence of some surgeons caused some complex injury of bile ducts.

- Patients should be transferred to hepato-biliary centers and well experienced surgeons.

- Intervention radiology procedures have a role in management of patients that developed stones over biliary enteric anastomosis.

- Intra-abdominal drain is preferred in all cases of laparoscopic cholecystectomy for at least 24 hours.

- Serious and immediate dealing with intraoperative injury of bile ducts.

- ERCP has a role by stent application and stone extraction from anastomotic site

- Roux-en-y hepatico-jeujonostomy is the preferred operative choice for managing BDI.

- Intervention radiology plays an important role in our study as PTD and balloon dilatation for patients with $\mathrm{H}-\mathrm{J}$ anastomotic stricture.

\section{References}

[1] U.Ahmad, Y.Jan, M.Uzair, Clinical presentation, types and treatment outcomes of Bile Duct Injuries (BDI) in laparoscopic cholecystectomy. Professional Medical Journal, Vol.27, PP.(2),2020.

[2] K.T.Buddingh, V.B.Nieuwenhuijs, L.van Buuren. Intraoperative assessment of biliary anatomy for prevention of bile duct injury: a review of current and future patient safety interventions. Surg Endosc, Vol. 25, PP.2449-2461,2015.

[3] P.Cantu, A.Tenca, C.Caparello, Role of symptoms, trend of liver tests and endotherapy in management of post-cholecystectomy biliary leak. Dig Dis Sci, Vol.56, PP.1565-1570,2010. 
[4] N.A.Hajjar, C.Tomus, L.Mocan, Management of bile duct injuries following laparoscopic cholecystectomy: long-term outcome and risk factors infuencing biliary reconstruction. Chirurgia (Bucur), Vol.109(4), PP.493-9,2014.

[5] S.Khanday, A.UI Hassan, Muneeb. The anatomical and surgical importance of hepatobiliary triangle of calot. International J.,Advanced Research, Vol.1(8), PP.856-858,2013.

[6] R.A.Kozar, M.K.McNutt. Management of adult blunt hepatic trauma. World J. Gastroenterology, Vol.16(6), PP.596-601,2016.

[7] Lee cooper, I.Giovannini, Review of introduction of laparoscope in general surgery. In: Blumgart L, editor. Surgery of the liver, biliary tract, and pancreas, vol. $1.4^{\text {th }}$ Edition Philadelphia: Saunders, Vol. ,pp3-29,2017.

[8] Med. Martin, Laparoscopic cholecystectomy, intra-operative cholangiography and common bile duct exploration. In: Mastery of Surgery. $3^{\text {rd }}$ ed. Vol. 2, Ch. 98. Ed. By: L.M. Nyhus; R.J. Baker and J.E. Fischer. Little, Brown and Company, London, New Boston and Toronto, Vol., PP.11001105. ,2017.

[9] H. H.Nasif, M.H.K.Abdelmaksoud, M.H.Shalaby. Role of Interventional Radiology in the Management of Postoperative Biliary Complications. The Egyptian J.,Hospital Medicine, Vol.72(9), PP. 5153-5160,2018.

[10] A.Paladini, A.Borzelli, D.B.Zobel, Corrigendum to "Combined surgical and interventional radiological treatment for biliary leakage following iatrogenic biliary obstruction'[Radiol
Case Rep 13 (2018) 772-777]. Radiology case reports, Vol. 14(7), PP. 897,2019.

[11]S.Sankar , K.Sreenivasan, Management of bile duct injury at various stages of presentation, Department of surgical gastroenterology, medical college and research institute, Vol. 600, PP.116 India,2012.

[12] G.E.I.Shallaly, A.Cuschieri, Nature, etiology and outcome of bile duct injuries after laparoscopic cholecystectomy. HPB Surg, Vol.2, PP.312,2000

[13] Sicklick, Retrospective studies of Bile duct injuries after laparoscopic cholecystectomy and post-operative advantages, 2015.

[14]A. Viste, A.Horn, K.Øvrebø. Bile duct injuries following laparoscopic cholecystectomy. Scandinavian J.,Surgery, Vol.104(4), PP.2337,2015.

[15] Y.Wang, Y.Liang, W.Wang. Management of electro thermal injury of common bile duct with a degradable biliary stent. Gastrointest Surg, Vol. 17, PP.1760-1765,2013.

[16] M.Wojcicki, W.Patkowski, T. Chmurowicz. Isolated right posterior bile duct injury following cholecystectomy. World J. Gastroenterology, Vol. 119, PP.6118-6121,2013.

[17] A.Paladini, A.Borzelli, D.B.Zobel. Corrigendum to "Combined surgical and interventional radiological treatment for biliary leakage following iatrogenic biliary obstruction" [Radiol Case Rep 13 (2018) 772-777]. Radiology case reports, Vol.14(7), PP. 897,2019. 